\title{
Bifurcation and Hybrid Control for A Simple Hopfield Neural Networks with Delays
}

\author{
Zisen Mao, Hao Wang, Dandan Xu, and Zhoujin Cui \\ College of Science, PLA University of Science and Technology, Nanjin 211101, China \\ Correspondence should be addressed to Zisen Mao; maozisen@126.com
}

Received 30 March 2013; Accepted 6 May 2013

Academic Editor: Guanghui Wen

Copyright (C) 2013 Zisen Mao et al. This is an open access article distributed under the Creative Commons Attribution License, which permits unrestricted use, distribution, and reproduction in any medium, provided the original work is properly cited.

A detailed analysis on the Hopf bifurcation of a delayed Hopfield neural network is given. Moreover, a new hybrid control strategy is proposed, in which time-delayed state feedback and parameter perturbation are used to control the Hopf bifurcation of the model. Numerical simulation results confirm that the new hybrid controller using time delay is efficient in controlling Hopf bifurcation.

\section{Introduction}

It is well known that neural networks are complex and largescale nonlinear dynamical system. In the last decade, the dynamical characteristics (including stable, unstable, oscillatory, and chaotic behavior) of Hopfield neural networks (HNNs) with time delays have become a subject of intense research activities. Many stability criteria are obtained. We refer the reader to [1-8] and the references cited therein. However, the periodic nature of neural impulses is of fundamental significance in the control of regular dynamical functions such as breathing and heart beating. Neural networks involving persistent oscillations such as limit cycle may be applied to pattern recognition and associative memory. In differential equations with delays, periodic oscillatory behavior can arise through the Hopf bifurcation. Therefore, it is also very significant to study the class of problem. Olien and Bélair [9] investigated the bifurcation of the following HNNs system:

$$
\begin{aligned}
\dot{x}_{1}= & -a_{1} x_{1}(t)+b_{11} f_{1}\left(x_{1}\left(t-\tau_{1}\right)\right) \\
& +b_{12} f_{2}\left(x_{2}\left(t-\tau_{2}\right)\right), \\
\dot{x}_{2}= & -a_{2} x_{2}(t)+b_{21} f_{1}\left(x_{1}\left(t-\tau_{3}\right)\right) \\
& +b_{22} f_{2}\left(x_{2}\left(t-\tau_{1}\right)\right),
\end{aligned}
$$

in which $\tau_{1}=\tau_{2}=\tau_{3}=\tau, a_{i}=1$ and $f_{i}(0)=0, i=1,2$, and Huang et al. [10] study further the bifurcation and periodic nature of system (1) with $2 \tau_{1}=\tau_{2}+\tau_{3}, a_{i}=1$ and $f_{i}(0)=0$, $i=1,2$. Moreover, many authors also consider discrete form of system (1); we can see [11-13].

In recent years, bifurcation control has attracted many researchers from various disciplines. The aim of bifurcation control is to design a controller to modify the bifurcation properties of a given nonlinear system, thereby to achieve some desirable dynamical behaviors. After the pioneering work initiated by Ott et al. [14], there have been many ideas and methods of bifurcation control [15-20]. However, from the control theory point of view, we may classify the current methods into two main categories: the first one is feedback control where state feedback is applied to control bifurcation or chaos, and the other is nonfeedback methods. Recently, Luo et al. [21] proposed a new control strategy for perioddoubling bifurcations in a discrete nonlinear dynamical system. Moreover, Liu and Chung [22] investigated the same control strategy in a continuous dynamical system without time delays. Now, we extend this strategy to deal with bifurcation control in HNNs system (1).

In the paper, we will propose a new hybrid control strategy in which the parameter perturbation and time-delayed state feedback are combined and used to control Hopf bifurcation in system (1). Simulation results demonstrate the correctness of our theoretical analysis. The comparison shows 
that the control strategy is effective as it meets the purpose of retarding the occurrence of bifurcation.

\section{Stability and Hopf Bifurcation of System (1) without Control}

In this section, we will consider system (1) with $2 \tau_{1}=\tau_{2}+$ $\tau_{3} \triangleq 2 \tau$ and $f_{i}(0)=0, i=1,2$. It is obviously that $(0,0)$ is an equilibrium point of system (1).

To simplify, here we denote $c_{11}=b_{11} f_{1}^{\prime}(0), c_{12}=b_{12} f_{2}^{\prime}(0)$, $c_{21}=b_{21} f_{1}^{\prime}(0), c_{22}=b_{22} f_{2}^{\prime}(0), a_{1}=a_{2}=b$. Consider the linearized system of system $(1)$ at $(0,0)$

$$
\begin{aligned}
& \dot{x}_{1}=-b x_{1}(t)+c_{11} x_{1}(t-\tau)+c_{12} x_{2}\left(t-\tau_{2}\right), \\
& \dot{x}_{2}=-b x_{2}(t)+c_{21} x_{1}\left(t-\tau_{3}\right)+c_{22} x_{2}(t-\tau) .
\end{aligned}
$$

The characteristic equation of the linearized system (2) is

$$
(\lambda+b)^{2}-\left(c_{11}+c_{22}\right)(\lambda+b) e^{-\lambda \tau}+\left(c_{11} c_{22}-c_{12} c_{21}\right) e^{-2 \lambda \tau}=0,
$$

which determines the local stability of the equilibrium solution. Thus, we will find some conditions which ensure that all roots of (3) have negative real parts. To facilitate the calculation in this paper, we rewrite the characteristic equation (3) as follows:

$$
(\lambda+b)^{2} e^{2 \lambda \tau}-2 T(\lambda+b) e^{\lambda \tau}+D=0
$$

where $D=c_{11} c_{22}-c_{12} c_{21}, T=(1 / 2)\left(c_{11}+c_{22}\right)$. Obviously, (4) is a quadratic polynomial in the variable $(\lambda+b) e^{\lambda \tau}$ and has roots given by

$$
(\lambda+b) e^{\lambda \tau}=T \pm \sqrt{T^{2}-D} .
$$

In the following, we distinguish two cases to discuss (5).

2.1. As $T^{2} \geq D$. In this part, we state a result due to [23] as a lemma to analyze (5), which is, for the convenience of the reader, stated as follows.

\section{Lemma 1. For the transcendental equation}

$$
\begin{aligned}
\lambda^{n} & +p_{1}^{(0)} \lambda^{n-1}+\cdots+p_{n-1}^{(0)} \lambda+p_{n}^{(0)} \\
& +\left[p_{1}^{(1)} \lambda^{n-1}+\cdots+p_{n-1}^{(1)} \lambda+p_{n}^{(1)}\right] e^{-\lambda \sigma_{1}} \\
& +\cdots+\left[p_{1}^{(m)} \lambda^{n-1}+\cdots+p_{n-1}^{(m)} \lambda+p_{n}^{(m)}\right] e^{-\lambda \sigma_{m}}=0,
\end{aligned}
$$

where $\sigma_{i} \geq 0(i=1, \ldots, m)$ and $p_{j}^{(i)}(i=1, \ldots, m ; j=$ $1, \ldots, n)$ are constants. As $\left(\sigma_{1}, \ldots, \sigma_{m}\right)$ varies, the sum of the orders of the zeros of (6) in the open right half-plane can change only if a zero appears on or crosses the imaginary axis.
For convenience, we make the following assumptions:

(H1) $b>T \pm \sqrt{T^{2}-D}$

(H2) $b^{2}>\left(T \pm \sqrt{T^{2}-D}\right)^{2}$.

(H3) $\left(T+\sqrt{T^{2}-D}\right)^{2}<b^{2}<\left(T-\sqrt{T^{2}-D}\right)^{2}$.

(H4) $b^{2}<\left(T \pm \sqrt{T^{2}-D}\right)^{2}$.

Lemma 2. If (H1) and (H2) hold, then all roots of (3) have negative real parts for every $\tau \in[0,+\infty)$.

Proof. For (3), when $\tau=0$, its roots can be expressed as $\lambda_{1,2}=-b+T \pm \sqrt{T^{2}-D}$. Clearly, all roots of (3) are negative if (H1) holds. We want to determine if the real part of some root increases to reach zero and eventually becomes positive as $\tau \neq 0$. We can see that $\lambda$ is a root of (3) if and only if $\lambda$ is a root of (5).

We write $\lambda=\rho+i \omega$ for a root of the characteristic equations (5), separate the real and imaginary parts of the ensuing equations (5), and obtain

$$
\begin{aligned}
& e^{\rho \tau}[(\rho+b) \cos (\omega \tau)-\omega \sin (\omega \tau)]=T \pm \sqrt{T^{2}-D} \\
& e^{\rho \tau}[(\rho+b) \sin (\omega \tau)+\omega \cos (\omega \tau)]=0
\end{aligned}
$$

A change in the stability of the stationary solution can only occur when $\rho=0$, that is,

$$
\begin{aligned}
& b \cos (\omega \tau)-\omega \sin (\omega \tau)=T \pm \sqrt{T^{2}-D}, \\
& b \sin (\omega \tau)+\omega \cos (\omega \tau)=0 .
\end{aligned}
$$

By (8), we have

$$
\omega^{2}=\left(T \pm \sqrt{T^{2}-D}\right)^{2}-b^{2} .
$$

By (9), if (H2) holds, we know that (5) has no purely imaginary roots, and then applying Lemma 1 one obtains that all roots of (3) have negative real parts. This completes the proof of lemma.

Lemma 3. For (5), one obtains the following results.

(1) If (H1) and (H3) hold, then (5) have a pair of purely imaginary roots $\pm i \omega_{-}$at $\tau=\tau_{-, j}$.

(2) If (H1) and (H4) hold, then (5) have a pair of purely imaginary roots $\pm i \omega_{+}$at $\tau=\tau_{+, j}$ and have another pair of purely imaginary roots $\pm i \omega_{-}$at $\tau=\tau_{-, j}$,

where

$$
\begin{gathered}
\omega_{ \pm}^{2}=\left(T \pm \sqrt{T^{2}-D}\right)^{2}-b^{2}, \\
\tau_{ \pm, j}=\frac{1}{\omega_{ \pm}} \arctan \left(-\frac{\omega_{ \pm}}{b}\right)+\frac{j \pi}{\omega_{ \pm}}, \quad j=0,1, \ldots ; \\
\tau_{0}=\min \left\{\tau_{+, 0}, \tau_{-, 0}\right\} .
\end{gathered}
$$

Here one denotes $\pm \omega_{0}$ especially as a pair of purely imaginary roots of (5) at $\tau=\tau_{0}$. To see if $\tau_{-, 0}$ and $\tau_{0}$ are bifurcation values, one needs to verify if the transversality conditions hold. In fact, one has the following. 
Lemma 4. The following transversality conditions:

$$
\left.\operatorname{Re}\left(\frac{d \lambda}{d \tau}\right)\right|_{\tau_{ \pm, j}}>0
$$

are satisfied.

Proof. By (5), we have

$$
\frac{d \lambda}{d \tau} e^{\lambda \tau}+(\lambda+b)\left(\tau e^{\lambda \tau} \frac{d \lambda}{d \tau}+\lambda e^{\lambda \tau}\right)=0
$$

Hence,

$$
\frac{d \lambda}{d \tau}(1+(\lambda+b) \tau)=-\lambda(\lambda+b)
$$

Obviously, we have

$$
\left(\frac{d \lambda}{d \tau}\right)^{-1}=\frac{(1+(\lambda+b) \tau)}{-\lambda(\lambda+b)}=-\frac{1}{\lambda(\lambda+b)}-\frac{\tau}{\lambda}
$$

then

$$
\begin{aligned}
\operatorname{sign}\left(\left.\operatorname{Re}\left(\frac{d \lambda}{d \tau}\right)\right|_{\tau_{ \pm, j}}\right) & =\operatorname{sign}\left(\left.\operatorname{Re}\left(\frac{d \lambda}{d \tau}\right)^{-1}\right|_{\tau_{ \pm, j}}\right) \\
& =\operatorname{sign}\left(\operatorname{Re}\left(\frac{-1}{i \omega(i \omega+b)}\right)\right) \\
& =\operatorname{sign}\left(\operatorname{Re}\left(\frac{-1}{-\omega^{2}+i b \omega}\right)\right) \\
& =\operatorname{sign}\left(\frac{-1 \cdot-\omega^{2}}{\omega^{4}+b^{2} \omega^{2}}\right) \\
& =\operatorname{sign}\left(\frac{1}{\omega^{2}+b^{2}}\right)>0 .
\end{aligned}
$$

We complete the proof of Lemma 4.

From Lemmas 2-4, we can obtain the following theorem about the distribution of the characteristic roots of (3).

Theorem 5. Let $\tau_{-, 0}, \tau_{0}$ be defined by (11).

(i) If (H1) and (H2) hold, then all roots of (3) have negative real parts for all $\tau \geq 0$.

(ii) If (H1) and $(H 3)((H 4))$ hold, then when $\tau<\tau_{-, 0}(\tau<$ $\left.\tau_{0}\right)$, all roots of (3) have negative real parts, when $\tau=$ $\tau_{-, 0}\left(\tau=\tau_{0}\right)$, (3) has a pair of purely imaginary roots $\pm i \omega_{-}\left( \pm i \omega_{0}\right)$, and when $\tau>\tau_{-, 0}\left(\tau>\tau_{0}\right)$, (3) has at least one root with positive real part.

By using Theorem 5, the stability and bifurcation of system (1) can be summarized as the following theorem.

Theorem 6. For system (1), let (H1) hold and let $\tau_{-, 0}, \tau_{0}$ be defined by (11).

(i) If (H2) holds, then the equilibrium point $(0,0)$ is asymptotically stable for discrete delays $\tau \geq 0$. (ii) If $(H 3)((H 4))$ holds, there is a critical value $\tau=$ $\tau_{-, 0}\left(\tau=\tau_{0}\right)$ of the discrete delay so that if $\tau<\tau_{-, 0}(\tau<$ $\left.\tau_{0}\right)$, then the equilibrium point $(0,0)$ is asymptotically stable; if $\tau>\tau_{-, 0}\left(\tau>\tau_{0}\right)$, then $(0,0)$ is unstable; Hopf bifurcation occurs when $\tau=\tau_{-, 0}\left(\tau=\tau_{0}\right)$.

2.2. As $T^{2}<D$. For convenience, we have the following assumptions:

(H5) $b>T$.

(H6) $b^{2}>D$.

(H7) $b^{2}<D$.

Similar to the deduction of Lemma 2, we have the following result.

Lemma 7. If (H5) and (H6) hold, then all roots of (3) have negative real parts for every $\tau \in[0,+\infty)$.

Proof. For (3), when $\tau=0$, its roots can be expressed as $\lambda_{1,2}=-b+T \pm \sqrt{T^{2}-D}$. Clearly, all roots of (3) have negative real parts if (H5) holds. We want to determine if the real part of some root increases to reach zero and eventually becomes positive as $\tau \neq 0$. We can see that $\lambda$ is a root of (3) if and only if $\lambda$ is a root of (5).

We write $\lambda=\rho+i \omega$ for a root of the characteristic equation (3), separate the real and imaginary parts of the ensuing equations (5), and obtain

$$
\begin{aligned}
& e^{\rho \tau}[(\rho+b) \cos (\omega \tau)-\omega \sin (\omega \tau)]=T, \\
& e^{\rho \tau}[(\rho+b) \sin (\omega \tau)+\omega \cos (\omega \tau)]= \pm \sqrt{D-T^{2}} .
\end{aligned}
$$

A change in the stability of the equilibrium point can only occur when $\rho=0$, that is,

$$
\begin{aligned}
& b \cos (\omega \tau)-\omega \sin (\omega \tau)=T, \\
& b \sin (\omega \tau)+\omega \cos (\omega \tau)= \pm \sqrt{D-T^{2}} .
\end{aligned}
$$

Hence, we have

$$
\omega^{2}=D-b^{2} .
$$

By (19), if (H6) holds, we know that (5) has no purely imaginary roots, and then applying Lemma 1 one obtains that all roots of (3) have negative real parts. This completes the proof of lemma.

Lemma 8. For (3), one obtains the following results.

If (H5) and (H7) hold, then (3) have a pair of purely imaginary roots $\pm i \omega$ at $\tau=\tau_{ \pm, j}$, where

$$
\omega^{2}=D-b^{2}
$$

$$
\tau_{ \pm, j}=\frac{1}{\omega} \arccos \left(\frac{b T \pm \omega \sqrt{D-T^{2}}}{b^{2}+\omega^{2}}\right)+\frac{2 j \pi}{\omega}, \quad j=0,1, \ldots ;
$$

$\tau_{0}=\min \left\{\tau_{+, 0}, \tau_{-, 0}\right\}$.

According to Lemma 4, one knows that $\tau_{0}$ is bifurcation values. 
From Lemmas 1 and 2, one can obtain the following theorem about the distribution of the characteristic roots of (3).

Theorem 9. Let $\tau_{0}$ be defined by (21).

(i) If (H5) and (H6) hold, then all roots of (3) have negative real parts for all $\tau \geq 0$.

(ii) If (H5) and (H7) hold, then when $\tau<\tau_{0}$, all roots of (3) have negative real parts, when $\tau=\tau_{0}$, (3) has a pair of purely imaginary roots $\pm i \omega$, and when $\tau>\tau_{0}$, (3) has at least one root with positive real part.

By using Theorem 9, the stability and bifurcation of system (1) can be summarized as the following theorem.

Theorem 10. For system (1), let (H5) hold and let $\tau_{0}$ be defined by the following: (21).

(i) If (H6) holds, then the equilibrium point $(0,0)$ is asymptotically stable for discrete delays $\tau \geq 0$.

(ii) If (H7) holds, there is a critical value $\tau=\tau_{0}$ of the discrete delay so that if $\tau<\tau_{0}$, then the equilibrium point $(0,0)$ is asymptotically stable; if $\tau>\tau_{0}$, then $(0,0)$ is unstable; Hopf bifurcation occurs when $\tau=\tau_{0}$.

\section{Stability Analysis and Bifurcation with Hybrid Control}

In this section, we will consider system (1) with hybrid control described by the following differential equation:

$$
\begin{aligned}
\dot{x}_{1}= & \alpha\left(-a_{1} x_{1}(t)+b_{11} f_{1}\left(x_{1}\left(t-\tau_{1}\right)\right)+b_{12} f_{2}\left(x_{2}\left(t-\tau_{2}\right)\right)\right) \\
& +\beta x_{1}\left(t-\tau_{1}\right), \\
\dot{x}_{2}= & \alpha\left(-a_{2} x_{2}(t)+b_{21} f_{1}\left(x_{1}\left(t-\tau_{3}\right)\right)+b_{22} f_{2}\left(x_{2}\left(t-\tau_{1}\right)\right)\right) \\
& +\beta x_{2}\left(t-\tau_{1}\right),
\end{aligned}
$$

where $\alpha>0$ and $\beta \in R$. Obviously, $(0,0)$ is also an equilibrium point of system (22).

Linearizing the system (22) at the equilibrium point $(0,0)$, we obtain

$$
\begin{aligned}
& \dot{x}_{1}=-\alpha b x_{1}(t)+\left(c_{11} \alpha+\beta\right) x_{1}\left(t-\tau_{1}\right)+c_{12} \alpha x_{2}\left(t-\tau_{2}\right), \\
& \dot{x}_{2}=-\alpha b x_{2}(t)+c_{21} \alpha x_{1}\left(t-\tau_{3}\right)+\left(c_{22} \alpha+\beta\right) x_{2}\left(t-\tau_{1}\right) .
\end{aligned}
$$

Then the characteristic equation for the linearized system around $(0,0)^{T}$ is given by

$$
\begin{gathered}
(\lambda+\alpha b)^{2} e^{2 \lambda \tau}-\left(\alpha\left(c_{11}+c_{22}\right)+2 \beta\right)(\lambda+\alpha b) e^{\lambda \tau} \\
+\left(\left(\alpha c_{11}+\beta\right)\left(\alpha c_{22}+\beta\right)-\alpha^{2} c_{12} c_{21}\right)=0,
\end{gathered}
$$

which is a quadratic polynomial in the variable $(\lambda+\alpha b) e^{\lambda \tau}$ and has roots given by

$$
(\lambda+\alpha b) e^{\lambda \tau}=T^{\prime} \pm \sqrt{\left(T^{\prime}\right)^{2}-D^{\prime}},
$$

where

$$
D^{\prime}=\alpha^{2} D+2 \alpha \beta T+\beta^{2}, \quad T^{\prime}=\alpha T+\beta .
$$

By (26), we know that $\left(T^{\prime}\right)^{2}-D^{\prime}=\alpha^{2}\left(T^{2}-D\right)$, and thus, $\left(T^{\prime}\right)^{2} \geq D^{\prime}\left(\left(T^{\prime}\right)^{2}<D^{\prime}\right)$ holds if and only if $T^{2} \geq D\left(T^{2}<D\right)$ holds.

In the following, we also distinguish two cases to discuss (25).

3.1. As $T^{2} \geq D$. Corresponding to Part I of Section 2, we make the following assumptions for convenience:

$$
\begin{aligned}
& (\mathrm{H} 1)^{\prime} b>T \pm \sqrt{T^{2}-D}+(\beta / \alpha) . \\
& (\mathrm{H} 2)^{\prime} b^{2}>\left(T \pm \sqrt{T^{2}-D}+(\beta / \alpha)\right)^{2} . \\
& (\mathrm{H} 3)^{\prime}\left(T+\sqrt{T^{2}-D}+(\beta / \alpha)\right)^{2}<b^{2}<\left(T-\sqrt{T^{2}-D}+(\beta / \alpha)\right)^{2} . \\
& (\mathrm{H} 4)^{\prime} b^{2}<\left(T \pm \sqrt{T^{2}-D}+(\beta / \alpha)\right)^{2} .
\end{aligned}
$$

Denote

$$
\begin{array}{r}
\left(\omega_{ \pm}^{\prime}\right)^{2}=\left(T^{\prime} \pm \sqrt{\left(T^{\prime}\right)^{2}-D^{\prime}}\right)^{2}-\alpha^{2} b^{2}, \\
\tau_{ \pm, j}^{\prime}=\frac{1}{\omega_{ \pm}^{\prime}} \arctan \left(-\frac{\alpha b}{\omega_{ \pm}}\right)+\frac{j \pi}{\omega_{ \pm}^{\prime}}, \quad j=0,1, \ldots, \\
\tau_{0}^{\prime}=\min \left\{\tau_{+, 0}^{\prime}, \tau_{-, 0}^{\prime}\right\} .
\end{array}
$$

Similarly, we can obtain the following theorem.

Theorem 11. For system (22), let $(H 1)^{\prime}$ hold and let $\tau_{ \pm, 0}^{\prime}$, $\tau_{0}^{\prime}$ be defined by (28).

(i) If $(\mathrm{H} 2)^{\prime}$ holds, then the equilibrium point $(0,0)$ is asymptotically stable for discrete delays $\tau \geq 0$.

(ii) If $(H 3)^{\prime}\left((H 4)^{\prime}\right)$ holds, there is a critical value $\tau=$ $\tau_{-, 0}^{\prime}\left(\tau=\tau_{0}^{\prime}\right)$ of the discrete delay so that if $\tau<\tau_{-, 0}^{\prime}(\tau<$ $\left.\tau_{0}^{\prime}\right)$, then the equilibrium point $(0,0)$ is asymptotically stable; if $\tau>\tau_{-, 0}^{\prime}\left(\tau>\tau_{0}^{\prime}\right)$, then $(0,0)$ is unstable; Hopf bifurcation occurs when $\tau=\tau_{-, 0}^{\prime}\left(\tau=\tau_{0}^{\prime}\right)$.

3.2. As $T^{2}<D$. Similar to deduction of Section 2.2, we have the following assumptions:

$(\mathrm{H} 5)^{\prime} b>T+(\beta / \alpha)$,

$(\mathrm{H} 6)^{\prime} b^{2}>D+(2 \beta / \alpha) T+(\beta / \alpha)^{2}$,

$(\mathrm{H} 7)^{\prime} b^{2}<D+(2 \beta / \alpha) T+(\beta / \alpha)^{2}$.

In this part, we denote

$$
\left(\omega^{\prime}\right)^{2}=D^{\prime}-\alpha^{2} b^{2},
$$

$$
\begin{aligned}
\tau_{ \pm, j}^{\prime}= & \frac{1}{\omega^{\prime}} \arccos \left(\frac{\alpha b T^{\prime} \pm \omega^{\prime} \sqrt{D^{\prime}-\left(T^{\prime}\right)^{2}}}{\alpha^{2} b^{2}+\left(\omega^{\prime}\right)^{2}}\right) \\
& +\frac{2 j \pi}{\omega^{\prime}}, \quad j=0,1, \cdots ; \tau_{0}^{\prime}=\min \left\{\tau_{+, j}^{\prime}, \tau_{-, j}^{\prime}\right\} .
\end{aligned}
$$

Hence, we can obtain the following theorem. 
Theorem 12. For system (22), let (H5)' hold and let $\tau_{0}^{\prime}$ be defined by (30).

(i) If (H6)' holds, then the equilibrium point $(0,0)$ is asymptotically stable for discrete delays $\tau \geq 0$.

(ii) If $(H 7)^{\prime}$ holds, there is a critical value $\tau=\tau_{0}^{\prime}$ of the discrete delay so that if $\tau<\tau_{0}^{\prime}$ then the equilibrium point $(0,0)$ is asymptotically stable; If $\tau>\tau_{0}^{\prime}$, then $(0,0)$ is unstable; Hopf bifurcation occurs when $\tau=\tau_{0}^{\prime}$.

Remark 13. When $\alpha=1-\gamma$ and $\beta=\gamma$ in the system (22), then we obtain the same hybrid control with [22]; however, a control model based on delayed feedback is proposed in this paper; it is well know that control theory should contain delay since any control action takes effect only after a certain delay. Hence, our hybrid control is more helpful than [22].

Remark 14. When $\alpha=1$ in the system (23), then we obtain a control model only based on delayed feedback in this paper, it is clear that our hybrid control is more general than control strategy proposed by [17].

Remark 15. In [10], authors investigated the Hopf bifurcation of following HNNs with $\alpha=1$ and $\beta=0$ :

$$
\begin{aligned}
\dot{x}_{1}=\alpha & \left(-x_{1}(t)-(\sqrt{2}-1) f_{1}\left(x_{1}\left(t-\tau_{1}\right)\right)\right. \\
& \left.+b_{12} f_{2}\left(x_{2}\left(t-\tau_{2}\right)\right)\right)+\beta x_{1}\left(t-\tau_{1}\right), \\
\dot{x}_{2}=\alpha & -x_{2}(t)+b_{21} f_{1}\left(x_{1}\left(t-\tau_{3}\right)\right) \\
& \left.-(\sqrt{2}-1) f_{2}\left(x_{2}\left(t-\tau_{1}\right)\right)\right)+\beta x_{2}\left(t-\tau_{1}\right),
\end{aligned}
$$

By choosing $\tau_{1}=3 \pi / 4, \tau_{2}=\pi / 8, \tau_{3}=11 \pi / 8$, the authors obtained that Hopf bifurcation occurs when $b_{12} b_{21}=1$. However, if choosing the parameters $\alpha=0.8$ and $\beta=0$, by the hybrid control strategy of this paper, the Hopf bifurcation in [10] will be eliminated. We can see Figures 1 and 2 .

Remark 16. It is known to all that neural networks are a special case of complex networks. Thus, it is interesting and important to further study how to expand the application of theoretical results in [24-27] and any other complex networks.

\section{Examples}

In this section, we give two examples to illustrate our results.

Example 1. Consider the following HNNs system with hybrid control:

$$
\begin{aligned}
\dot{x}_{1}= & \alpha\left(-a_{1} x_{1}(t)+b_{11} f_{1}\left(x_{1}\left(t-\tau_{1}\right)\right)+b_{12} f_{2}\left(x_{2}\left(t-\tau_{2}\right)\right)\right) \\
& +\beta x_{1}\left(t-\tau_{1}\right), \\
\dot{x}_{2}= & \alpha\left(-a_{2} x_{2}(t)+b_{21} f_{1}\left(x_{1}\left(t-\tau_{3}\right)\right)+b_{22} f_{2}\left(x_{2}\left(t-\tau_{1}\right)\right)\right) \\
& +\beta x_{2}\left(t-\tau_{1}\right),
\end{aligned}
$$
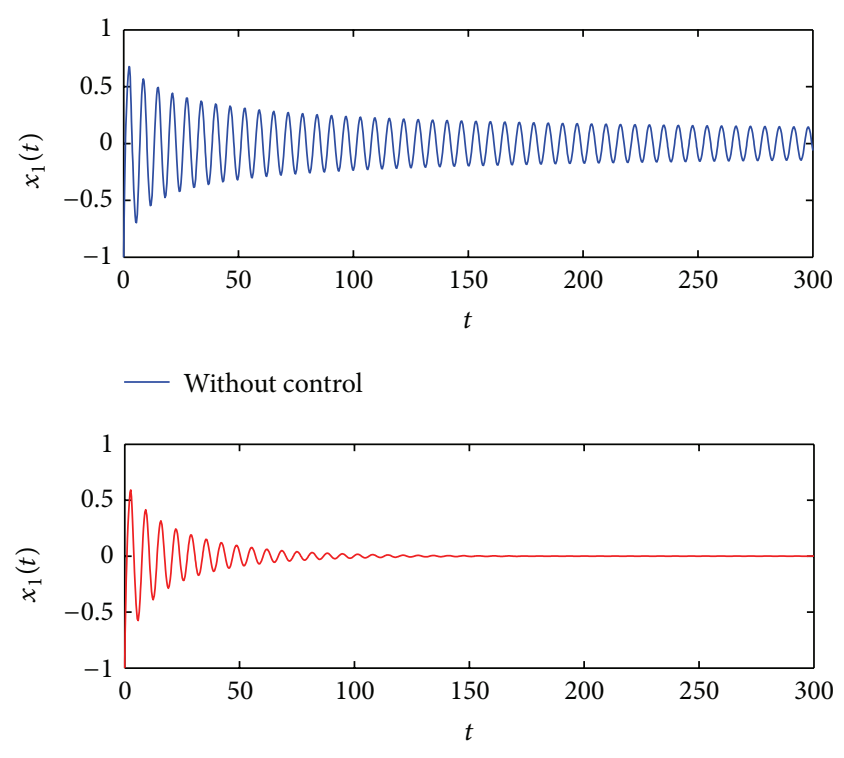

— With control

FIGURE 1: The trajectory of $x_{1}(t)$ versus time in the system (31) with $\tau=3 \pi / 4$.

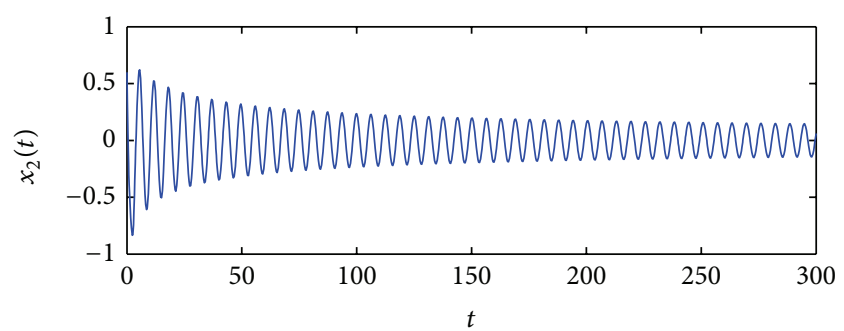

Without control

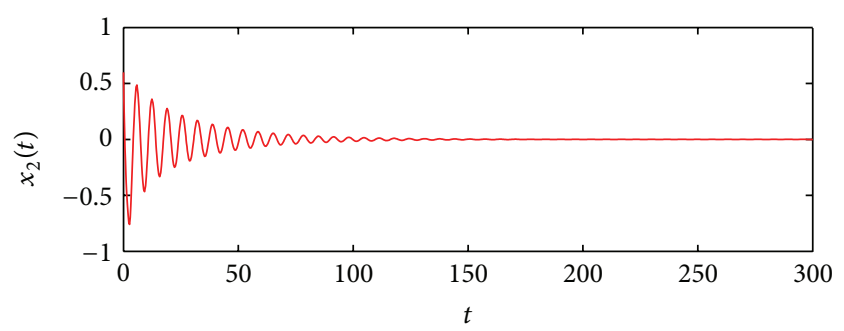

— With control

FIGURE 2: The trajectory of $x_{2}(t)$ versus time in the system (31) with $\tau=3 \pi / 4$.

where $\tau_{1}=\tau_{2}=\tau_{3}=\tau, a_{i}=0.5, b_{i j}=-0.3$, and $f_{i}(x)=$ $\tanh (x), i, j=1,2$. It is obvious that $(0,0)$ is an equilibrium point of system (32). Choosing $\alpha=1, \beta=0$, by calculation, the periodic oscillatory behavior can arise through the Hopf bifurcation as $\tau_{0}=7.7063$; we can see Figures 3 and 5 ( $\tau=7.7063, \tau=17.7063)$. However, when $\alpha / 5<2 \beta<\alpha$, with complicated calculation, (H2)' holds; by Theorem 11(i), the equilibrium point $(0,0)$ is asymptotically stable for any discrete delays $\tau \geq \tau_{0}$. For the convenience of numerical 


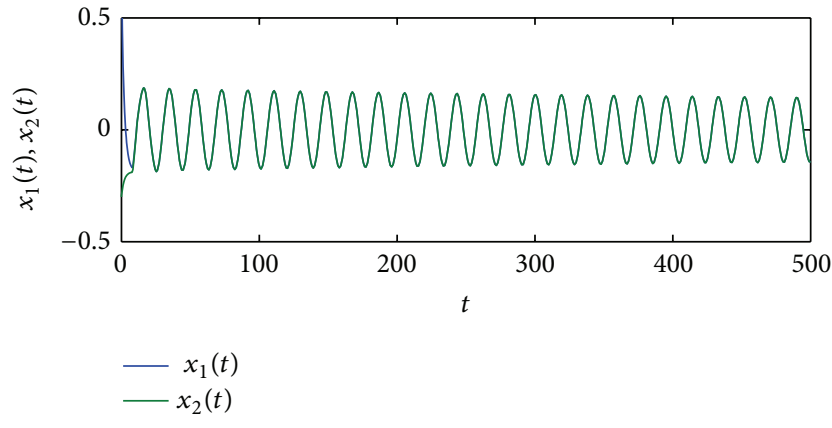

FIGURE 3: The trajectory of $x_{1}(t)$ and $x_{2}(t)$ versus time in the system (32) without control $(\tau=7.7063)$.

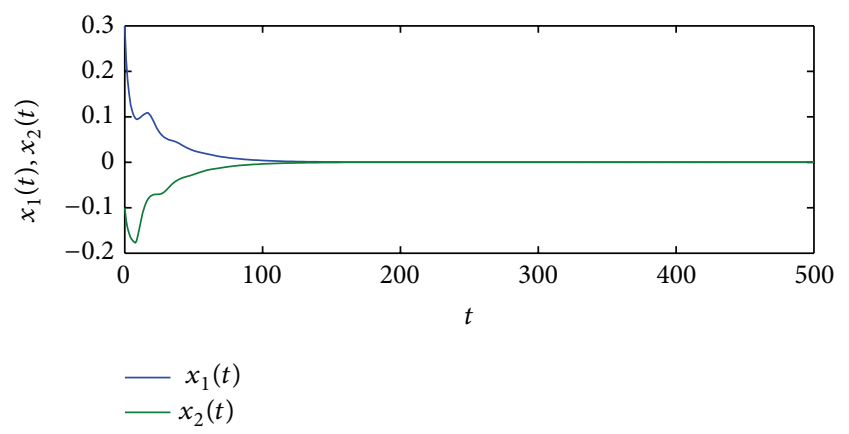

FIGURE 4: The trajectory of $x_{1}(t)$ and $x_{2}(t)$ versus time in the system (32) with control $(\tau=7.7063)$.

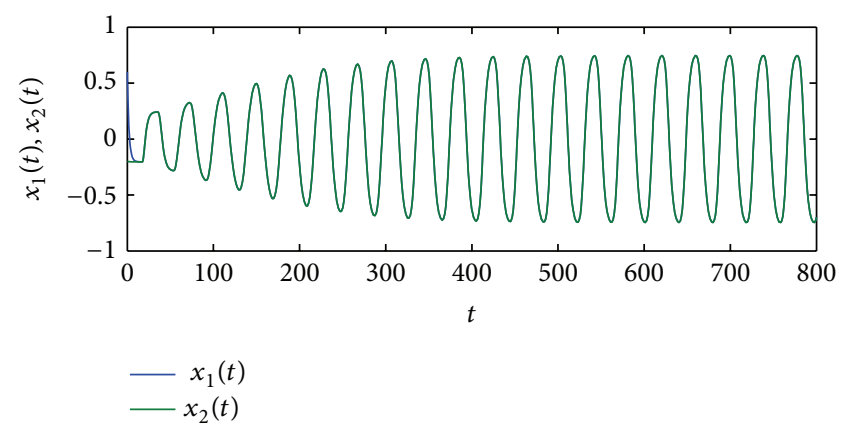

FIGURE 5: The trajectory of $x_{1}(t)$ and $x_{2}(t)$ versus time in the system (32) without control $(\tau>7.7063)$.

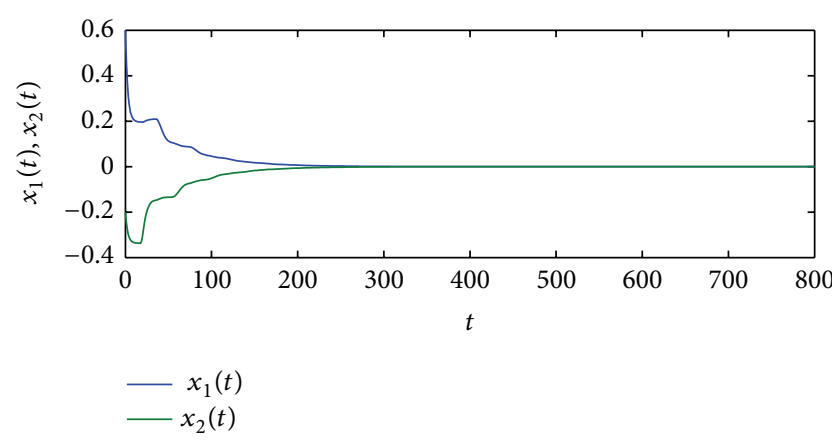

FIgURE 6: The trajectory of $x_{1}(t)$ and $x_{2}(t)$ versus time in the system (32) with control $(\tau>7.7063)$.

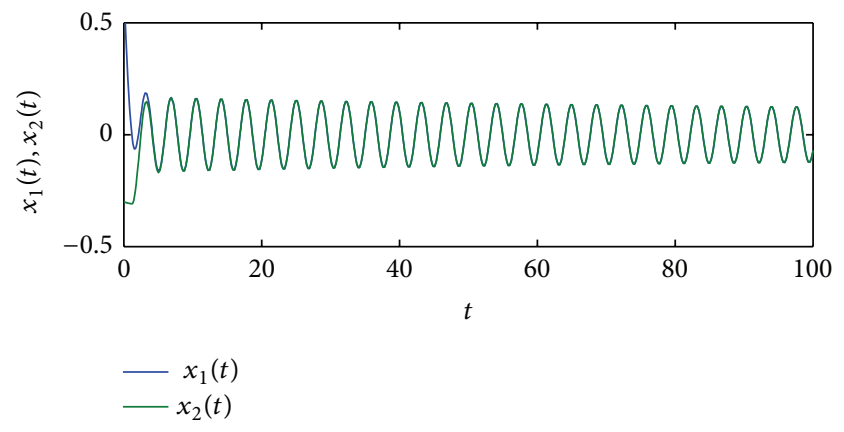

FIGURE 7: The trajectory of $x_{1}(t)$ and $x_{2}(t)$ versus time in the system (33) without control $(\tau=1.2092)$.

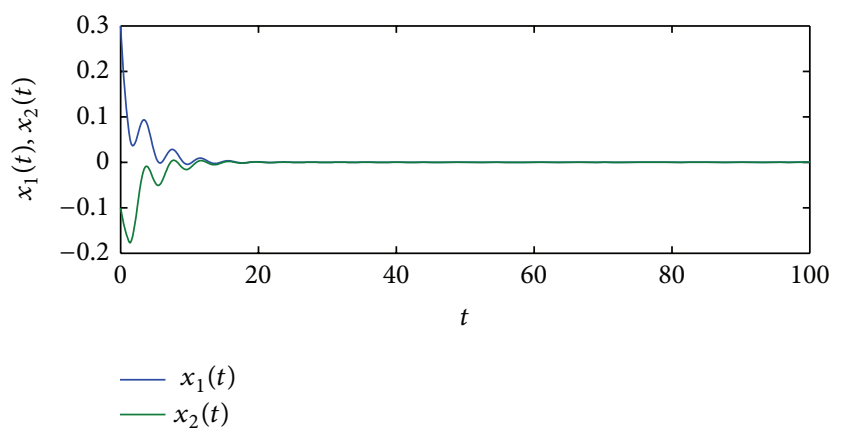

FIGURE 8: The trajectory of $x_{1}(t)$ and $x_{2}(t)$ versus time in the system (33) with control $(\tau=1.2092)$.

simulation, here we choose $\alpha=0.75, \beta=0.25$, and $\tau=$ 17.7063 as an example. it can be seen in Figure $4(\tau=7.7063)$. Fix all coefficients of system (32) and let $\tau$ vary, and the waveforms $x_{1}(t), x_{2}(t)$ without and with control are shown, respectively. Obviously, we obtain that the Hopf bifurcation in (32) without hybrid control could be eliminated by hybrid control; we can see Figure $6\left(\tau=17.7063>\tau_{0}\right)$.

Example 2. Consider the following HNNs system with hybrid control:

$$
\begin{aligned}
\dot{x}_{1}= & \alpha\left(-a_{1} x_{1}(t)+b_{11} f_{1}\left(x_{1}\left(t-\tau_{1}\right)\right)+b_{12} f_{2}\left(x_{2}\left(t-\tau_{2}\right)\right)\right) \\
& +\beta x_{1}\left(t-\tau_{1}\right), \\
\dot{x}_{2}= & \alpha\left(-a_{2} x_{2}(t)+b_{21} f_{1}\left(x_{1}\left(t-\tau_{3}\right)\right)+b_{22} f_{2}\left(x_{2}\left(t-\tau_{1}\right)\right)\right) \\
& +\beta x_{2}\left(t-\tau_{1}\right),
\end{aligned}
$$

where $\tau_{1}=\tau_{2}=\tau_{3}=\tau, a_{i}=1, b_{i j}=-1$, and $f_{i}(x)=\tanh (x)$, $i, j=1,2$. It is obvious that $(0,0)$ is an equilibrium point of system (33). Choosing $\alpha=1, \beta=0$, by calculation, we know $\tau_{0}=1.2092$. However, when $-\alpha<\beta<\alpha$, a family of periodic solutions bifurcates from $(0,0)$ at $\tau_{0}^{\prime}$ (see Figure 7 ). Choosing $\alpha=0.75$ and $\beta=0.25$, with complicated calculation, we know $\tau_{0}^{\prime}=2.2143$ (see Figure 8 ). Fix all coefficients of system (33) and let $\tau=\tau_{0}$; the waveforms $x_{1}(t), x_{2}(t)$ without and with control are shown, respectively. However, the Hopf 


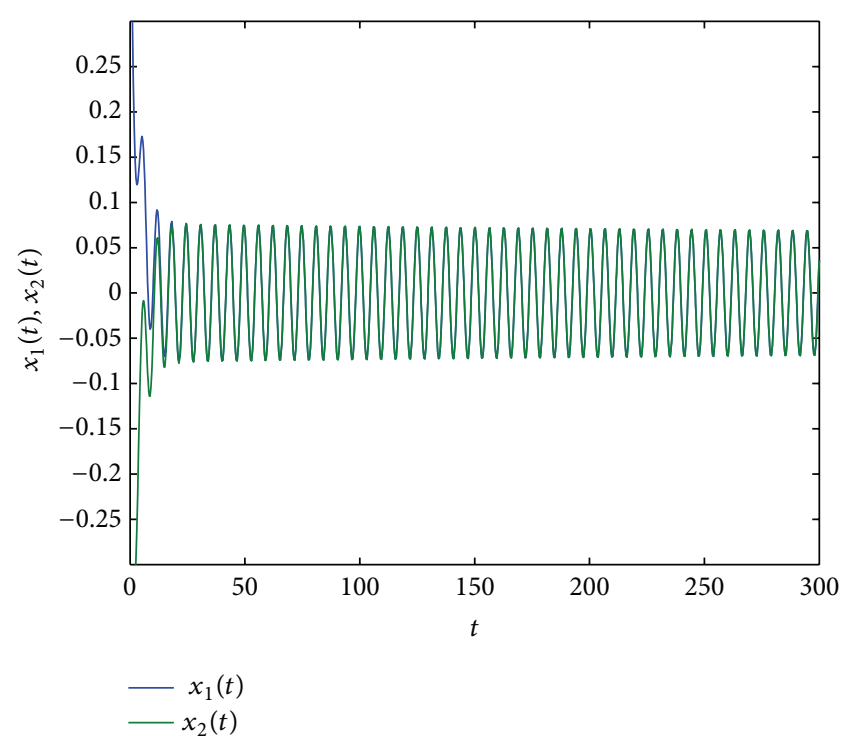

FIGURE 9: The trajectory of $x_{1}(t)$ and $x_{2}(t)$ versus time in the system (33) with control $(\tau=2.2143)$.

bifurcation in (33) could be delayed by hybrid control which could be seen by Figure 9.

\section{Conclusions}

In this paper, the bifurcation and the bifurcation control problems have further been investigated for a HNNs model with delays. For the model, hybrid control strategy in which the parameter perturbation and time-delayed state feedback are combined and used to control various bifurcations in a continuous nonlinear dynamical system. It should be pointed out that, although Liu also have dealt with hybrid control, the time delayed feedback control used in our paper is more helpful than the controller in [22]. On the other hand, using parameter perturbation in this paper, our control strategy is more general than the other feedback control. Numerical simulations are given to justify the validity of hybrid controller in bifurcation control.

\section{Acknowledgments}

This research was supported by the Pre-research Foundation of PLA University of Science and Technology. Youth Research Foundation of College of Science of PLA University of Science and Technology.

\section{References}

[1] J. J. Hopfield, "Neurons with graded response have collective computational properties like those of two-state neurons," Proceedings of the National Academy of Sciences of the United States of America, vol. 81, no. 10, pp. 3088-3092, 1984.

[2] C. Li, X. Liao, and K.-W. Wong, "Delay-dependent and delayindependent stability criteria for cellular neural networks with delays," International Journal of Bifurcation and Chaos in
Applied Sciences and Engineering, vol. 16, no. 11, pp. 3323-3340, 2006.

[3] V. Singh, "On global robust stability of interval Hopfield neural networks with delay," Chaos, Solitons and Fractals, vol. 33, no. 4, pp. 1183-1188, 2007.

[4] L. Wan and J. Sun, "Mean square exponential stability of stochastic delayed Hopfield neural networks," Physics Letters A, vol. 343, no. 4, pp. 306-318, 2005.

[5] Z. Wang, Y. Liu, K. Fraser, and X. Liu, "Stochastic stability of uncertain Hopfield neural networks with discrete and distributed delays," Physics Letters A, vol. 354, no. 4, pp. 288-297, 2006.

[6] D.-Y. Xu and H.-Y. Zhao, "Invariant and attracting sets of Hopfield neural networks with delay," International Journal of Systems Science, vol. 32, no. 7, pp. 863-866, 2001.

[7] Z. Yuan, D. Hu, L. Huang, and G. Dong, "On the global asymptotic stability analysis of delayed neural networks," International Journal of Bifurcation and Chaos in Applied Sciences and Engineering, vol. 15, no. 12, pp. 4019-4025, 2005.

[8] H. Zhao, "Global asymptotic stability of Hopfield neural network involving distributed delays," Neural Networks, vol. 17, no. 1, pp. 47-53, 2004.

[9] L. Olien and J. Bélair, "Bifurcations, stability, and monotonicity properties of a delayed neural network model," Physica D, vol. 102, no. 3-4, pp. 349-363, 1997.

[10] C. Huang, Y. He, L. Huang, and Y. Zhaohui, "Hopf bifurcation analysis of two neurons with three delays," Nonlinear Analysis. Real World Applications, vol. 8, no. 3, pp. 903-921, 2007.

[11] W. He and J. Cao, "Stability and bifurcation of a class of discretetime neural networks," Applied Mathematical Modelling, vol. 31, no. 10, pp. 2111-2122, 2007.

[12] Z. Yuan, D. Hu, and L. Huang, "Stability and bifurcation analysis on a discrete-time system of two neurons," Applied Mathematics Letters, vol. 17, no. 11, pp. 1239-1245, 2004.

[13] H. Zhao, L. Wang, and C. Ma, "Hopf bifurcation and stability analysis on discrete-time Hopfield neural network with delay," Nonlinear Analysis. Real World Applications, vol. 9, no. 1, pp. 103-113, 2008.

[14] E. Ott, C. Grebogi, and J. A. Yorke, “Controlling chaos," Physical Review Letters, vol. 64, no. 11, pp. 1196-1199, 1990.

[15] E. H. Abed, H. O. Wang, and R. C. Chen, "Stabilization of period doubling bifurcations and implications for control of chaos," Physica D, vol. 70, no. 1-2, pp. 154-164, 1994.

[16] Y. Braiman and I. Goldhirsch, "Taming chaotic dynamics with weak periodic perburbations," Physical Review Letters, vol. 66, no. 20, pp. 2545-2548, 1991.

[17] G. Chen, "On some controllability conditions for chaotic dynamics control," Chaos, Solitons and Fractals, vol. 8, no. 9, pp. 1461-1470, 1997.

[18] R. Lima and M. Pettini, "Suppression of chaos by resonant parametric perturbations," Physical Review A, vol. 41, no. 2, pp. 726733, 1990.

[19] X. F. Wang and G. Chen, "Pinning control of scale-free dynamical networks," Physica A, vol. 310, no. 3-4, pp. 521-531, 2002.

[20] L. Yang, Z. Liu, and J. Mao, “Controlling hyperchaos," Physical Review Letters, vol. 84, pp. 67-70, 2000.

[21] X. S. Luo, G. Chen, B. H. Wang, and J. Q. Fang, "Hybrid control of period-doubling bifurcation and chaos in discrete nonlinear dynamical systems," Chaos, Solitons and Fractals, vol. 18, no. 4, pp. 775-783, 2003. 
[22] Z. Liu and K. W. Chung, "Hybrid control of bifurcation in continuous nonlinear dynamical systems," International Journal of Bifurcation and Chaos in Applied Sciences and Engineering, vol. 15, no. 12, pp. 3895-3903, 2005.

[23] S. Ruan and J. Wei, "On the zeros of transcendental functions with applications to stability of delay differential equations with two delays," Dynamics of Continuous, Discrete \& Impulsive Systems A, vol. 10, no. 6, pp. 863-874, 2003.

[24] W. Yu and J. Cao, "Synchronization control of stochastic delayed neural networks," Physica A, vol. 373, pp. 252-260, 2007.

[25] W. Yu, G. Chen, and J. Lü, "On pinning synchronization of complex dynamical networks," Automatica, vol. 45, no. 2, pp. 429435, 2009.

[26] G. Wen, Z. Duan, W. Yu, and G. Chen, "Consensus in multiagent systems with communication constraints," International Journal of Robust and Nonlinear Control, vol. 22, no. 2, pp. 170182, 2012.

[27] G. Wen, Z. Duan, W. Yu, and G. Chen, "Consensus of secondorder multi-agent systems with delayed nonlinear dynamics and intermittent communications," International Journal of Control, vol. 86, no. 2, pp. 322-331, 2013. 


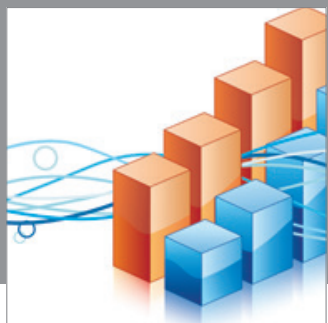

Advances in

Operations Research

mansans

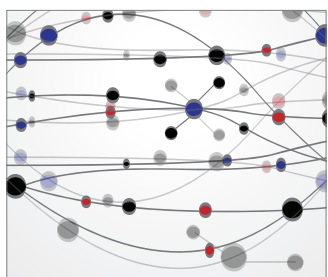

The Scientific World Journal
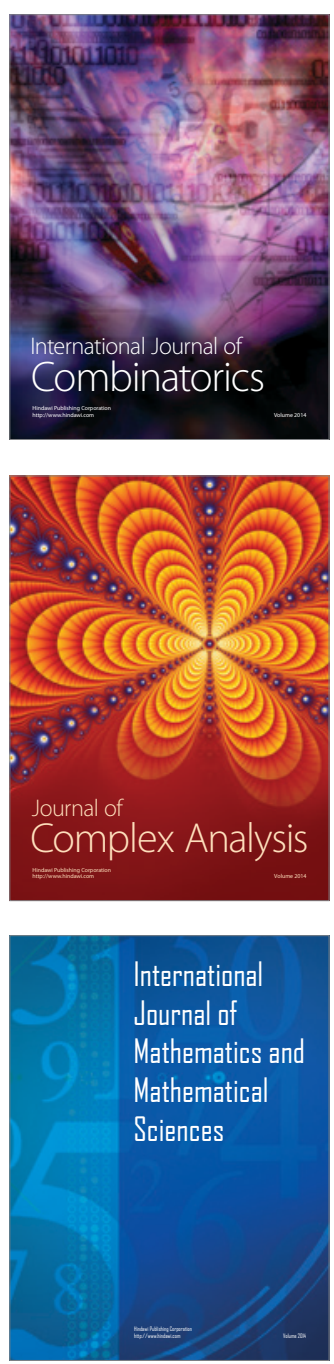
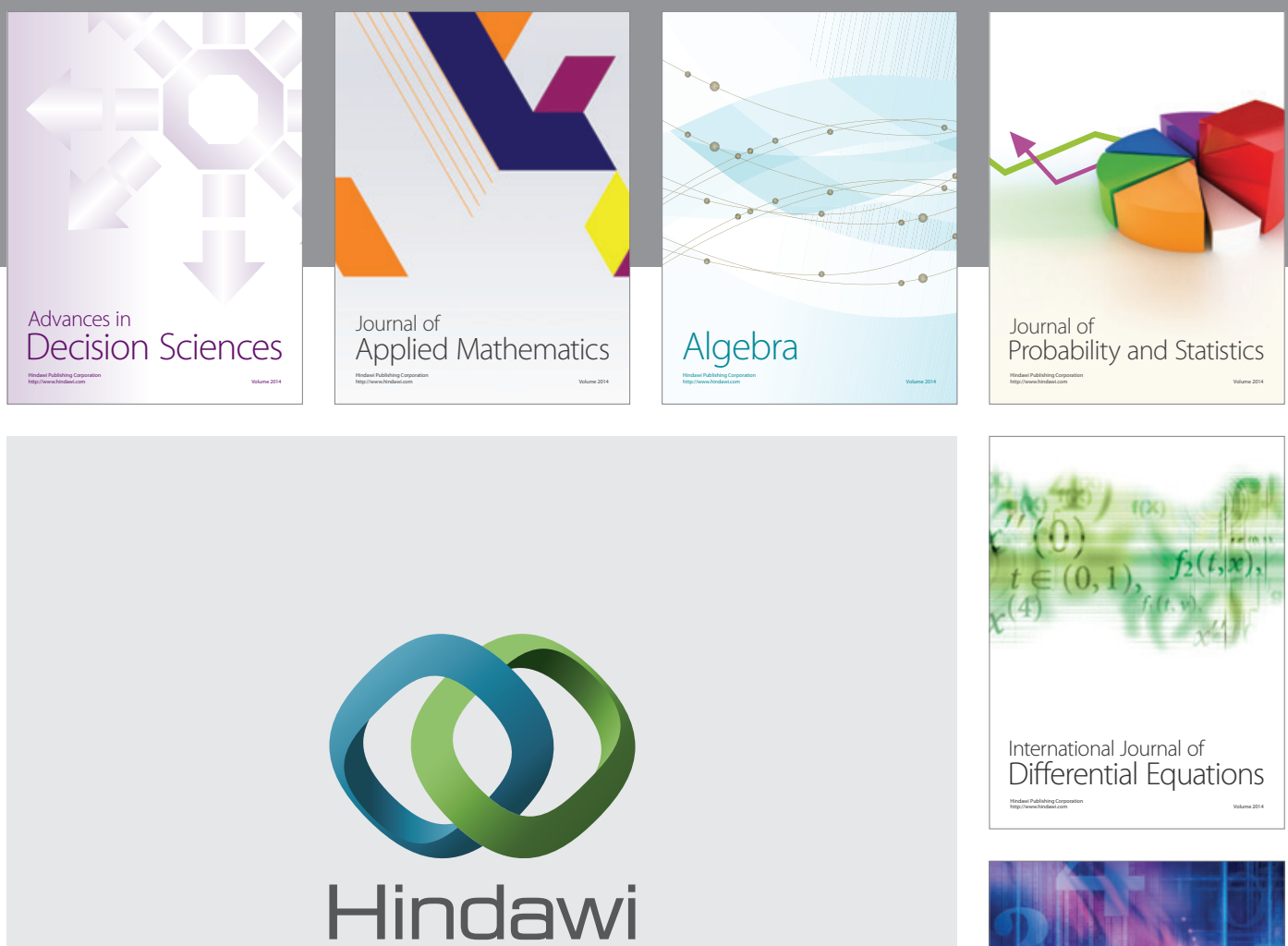

Submit your manuscripts at http://www.hindawi.com
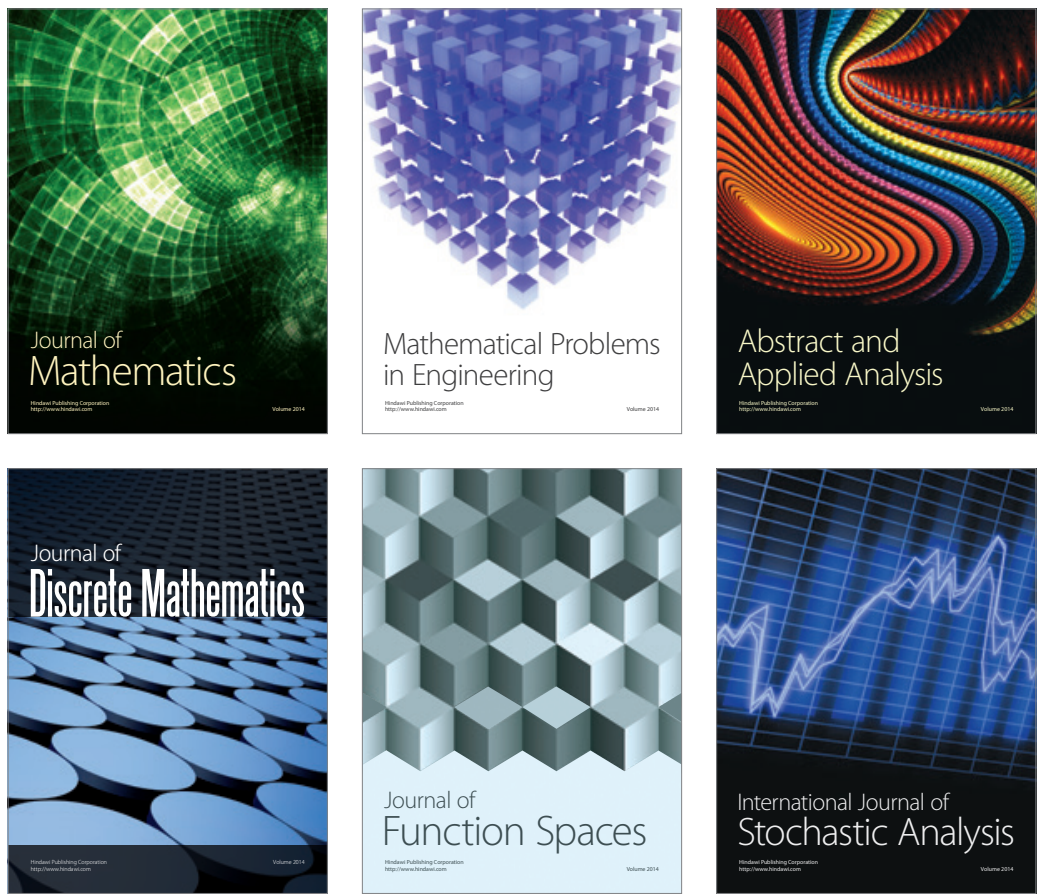

Journal of

Function Spaces

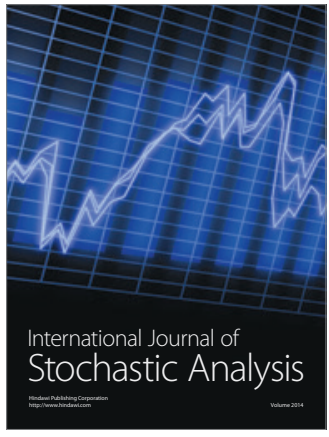

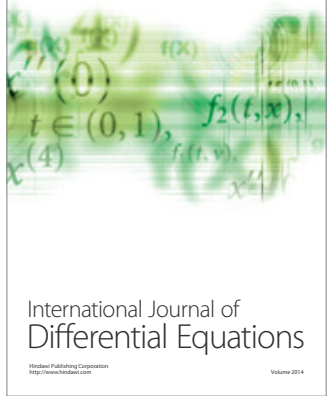
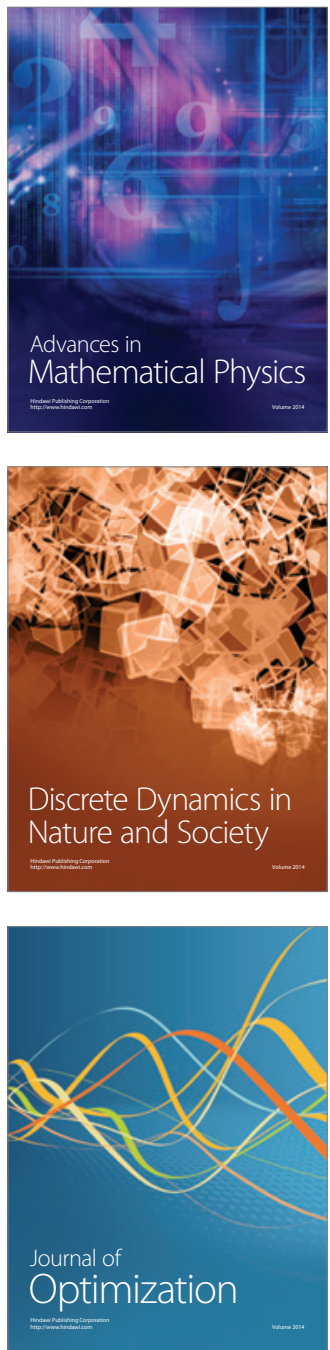